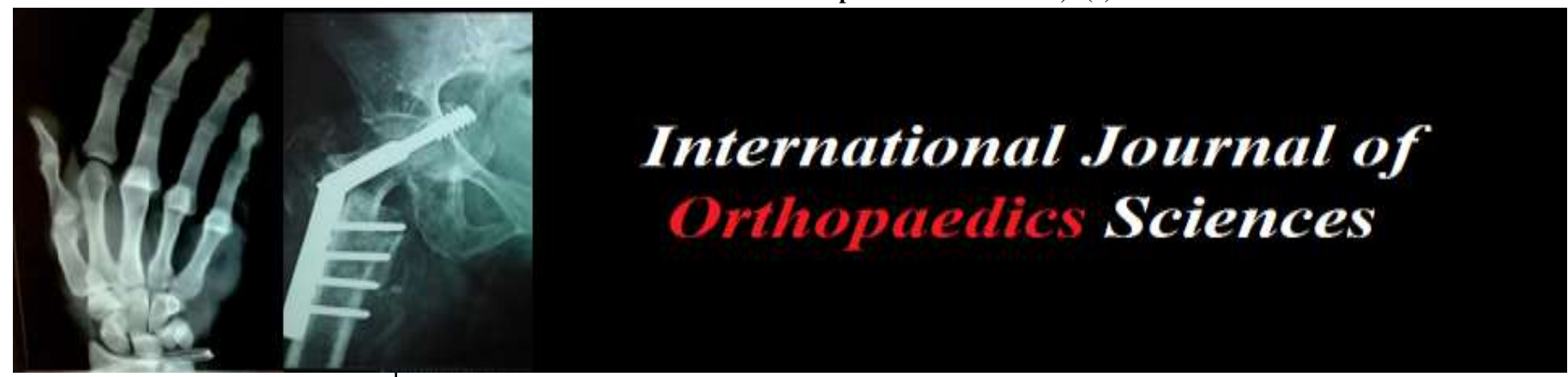

E-ISSN: 2395-1958

P-ISSN: 2706-6630

IJOS 2021; 7(3): 108-115

(C) 2021 IJOS

www.orthopaper.com

Received: 22-04-2021

Accepted: 27-06-2021

Kavita Avinash Patil

Research Scholar, Department of

Electronics \& Communication

Engineering, SJB Institute of

Technology (Sri

Adichunchanagiri Shikshana

Trust), Faculty, East Point

College of Engineering and

Technology, Affiliated to

Visvesvaraya Technological

University, Bangalore,

Karnataka, India

KV Mahendra Prashanth Professor, Department of

Electronics and Communication

Engg SJB Institute of

Technology, Affiliated to

Visvesvaraya Technological

University, Bangalore,

Karnataka, India

Dr. A Ramalingaiah

M.S (Ortho) Orthopedic Doctor

SJB Institute of Technology,

Affiliated to Visvesvaraya

Technological University,

Bangalore, Karnataka, India

Corresponding Author:

Kavita Avinash Patil

Research Scholar, Department of

Electronics\& Communication

Engineering, SJB Institute of

Technology (Sri

Adichunchanagiri Shikshana

Trust), Faculty, East Point

College of Engineering and

Technology, Affiliated to

Visvesvaraya Technological

University, Bangalore,

Karnataka, India

\section{A comparative study on detection of osteoporosis using deep learning methods: A review}

\section{Kavita Avinash Patil, KV Mahendra Prashanth and Dr. A Ramalingaiah}

DOI: https://doi.org/10.22271/ortho.2021.v7.i3b.2736

\section{Abstract}

Osteoporosis is a silent bone disease characterized by low bone mass and loss of bone tissue that may lead to weak and fragile bones and decreases in bone strength which increases the risk of fractures.It is more common in women rather than men. DEXA that is Dual Energy X-ray Absorptiometry is a model to diagnose osteoporosis although its low-availability, expensive, and high radiation exposure. The CAD (Computer-Aided Diagnosis) has enhanced the analysis to a higher level. The advanced learning paradigm that is Deep-Learn, Machine-Learn and Artificial Intelligence has exposed a turning point in the medical field which leads to accurate diagnosis of osteoporosis. The review is based on various anatomical sites such as lumbar spine, hip, forearm, calcaneus, and dental are assisted and examined based on validation, pre-trained networks, and accuracy. The combination of clinical data and images are fed to deep leaning models specifically CNN-Convolutional Neural Network, RNN-Recurrent Neural Network may result in completely automatic detection and diagnosis of osteoporosis.

Keywords: Artificial intelligence, classification, deep learning, feature extraction, machine learning, osteoporosis

\section{Introduction}

Osteoporosis means porous bone, identified as a disease categorized as a micro architectural deterioration of bone tissue and low bone mass which leads to brittleness bone, increases the risk fractures of the wrist, hip, and spine ${ }^{[1]}$. In Indian women Osteoporosis is a major public health issue, expected over the age of 50 years, postmenopausal relates to bone metabolism which increases the rates of fractures. The bones of the spine affected by osteoporosis of vertebrae which in turn leads to hunched or stooped posture. The fracture rate can be reduced by the intake of calcium and vitamin $\mathrm{D}^{[2]}$. The decreasing bone-mass with age in men and women as shown in Fig 1.

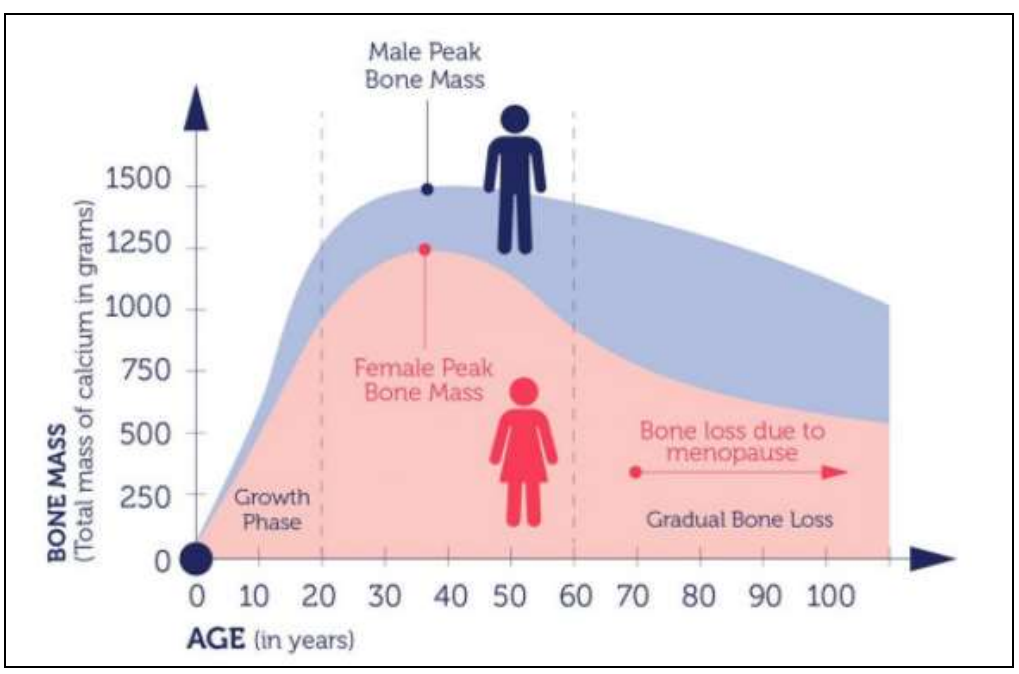

Fig 1: Decreasing bone-mass with age in men and women ${ }^{[28]}$ 
Artificial Intelligence (AI) has the potential of a machine to mimic human behaviour intelligence-is perched to transform the medical practice ${ }^{[3]}$. AI is a compatible technology, designed to develop the human performances of medical researchers, physicians, and nurses. In medicine, AI is not to substitute the doctor but to intensify medical expertise. In recent times, $\mathrm{AI}$ is a hot issue of the 4th industrial revolt. The approaches of AI tool trained to diagnose the patients with fracture which leads to the risk of osteoporosis precede the radiologist traditionally examine the report. The fig. 2 shows the stages of the Intelligent System.
Machine learning (ML) is a cluster of artificial intelligence that creates healthcare smarter and tool for medical research like diagnostic imaging, treatment optimization, genetic tests, and electro diagnosis. The mainstay task of ML is classifier and prediction. Its role is applied in the field of medicine, which helps to diagnose and treat patients. Depending on the prediction utilization of ML is developed to assess the T-score of the lumbar spine and categorize the healthy vertebra and osteoporotic vertebrae by the usage of Hounsfield units (HU) of lumbar computed-tomography (CT) ${ }^{[4]}$.

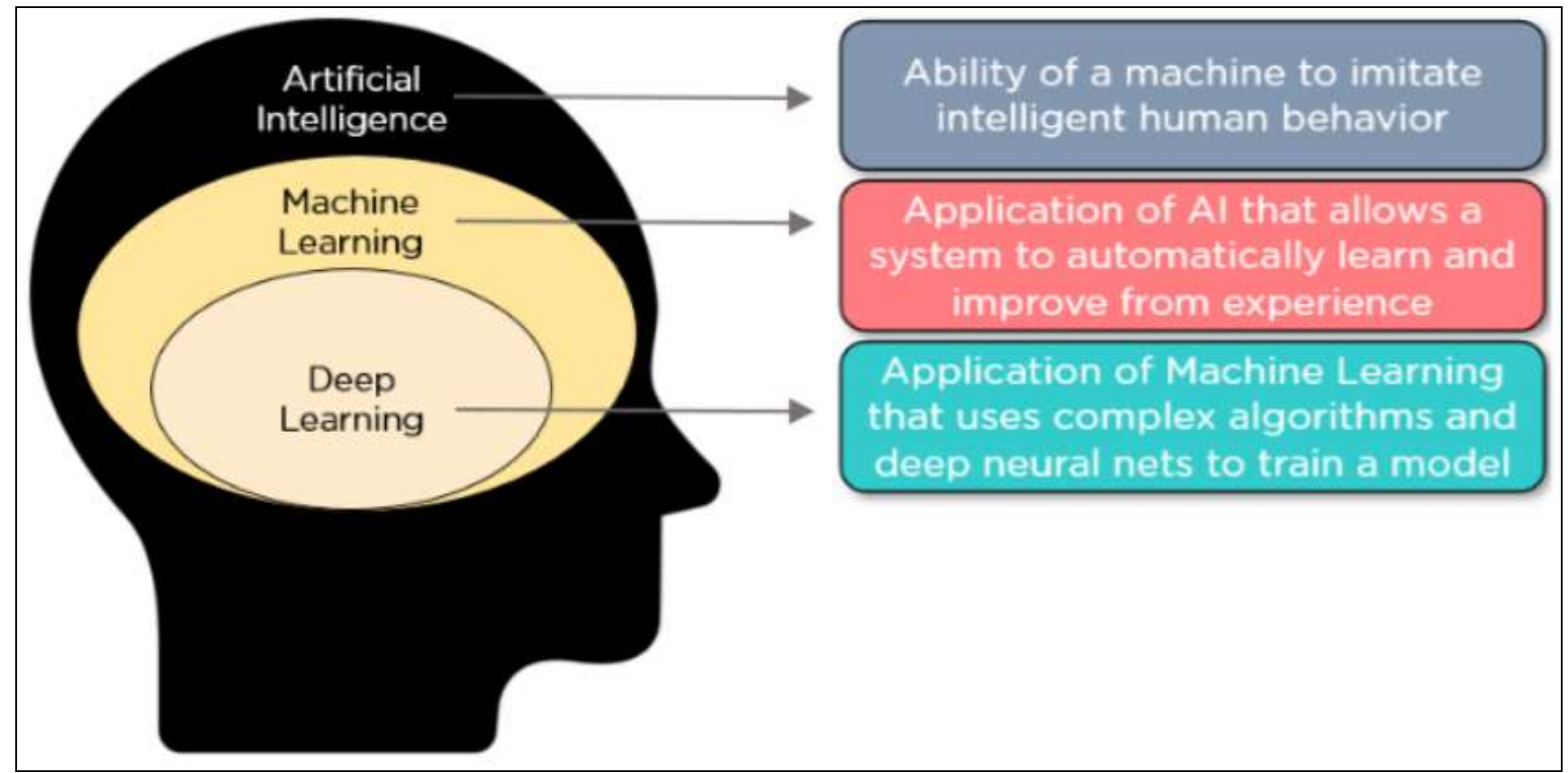

Fig 2: Intelligent System (Artificial Intelligence, Machine learning and Deep learning) ${ }^{\text {29] }}$

ML has ample scope in medical use will be stable and incessant which is essential for a doctor to gain the fundamental idea of ML. The major categories of ML are supervised and unsupervised learning. In Supervised learning (SL) the datasets are labelled and trained to predict the output from the input data and in unsupervised learning, the datasets are not labelled and be trained to inherit itself from the existing data ${ }^{[5]}$. The logistic and linear regression is a part of the SL algorithm which is used as a predictive paradigm. The Linear regression (LR) patterns show to predict the linear relationship between two variables (dependent and independent) and this pattern correlates the independent (sex, age, and HU of lumbar CT) and dependent (T-score) variables and logistic regression (LoR). is a type of binary regression where dependent variable has only two (" 0 " and " 1 ") values i.e., output is classified as the controlled and osteoporotic spine .To design a model in the medical field, it is essential to comprehend the fundamental concept of ML and AI and to improve accuracy.

The methodology used in deep learning use architecture of the neural network, the "deep" indicates an increase in numeral hidden layers hence the models are named as a deep neural network (DNN). Conventional the approach in neural network (NN) have two-three hidden layers but DNN has innumerable as 150. DNN uses large labelled datasets while NN doesn't require manual attribute extraction as it learns directly the features from the data ${ }^{[7]}$. The current trend in DNN is the Convolutional neural network (ConvNet or CNN). It directly extracts the features from image because CNN does convolution between input data and learned features and uses two dimensional (2-D) Convolutional layers which makes architecture processing compatible with 2-D image data. The automatic extraction of features improves accuracy. 


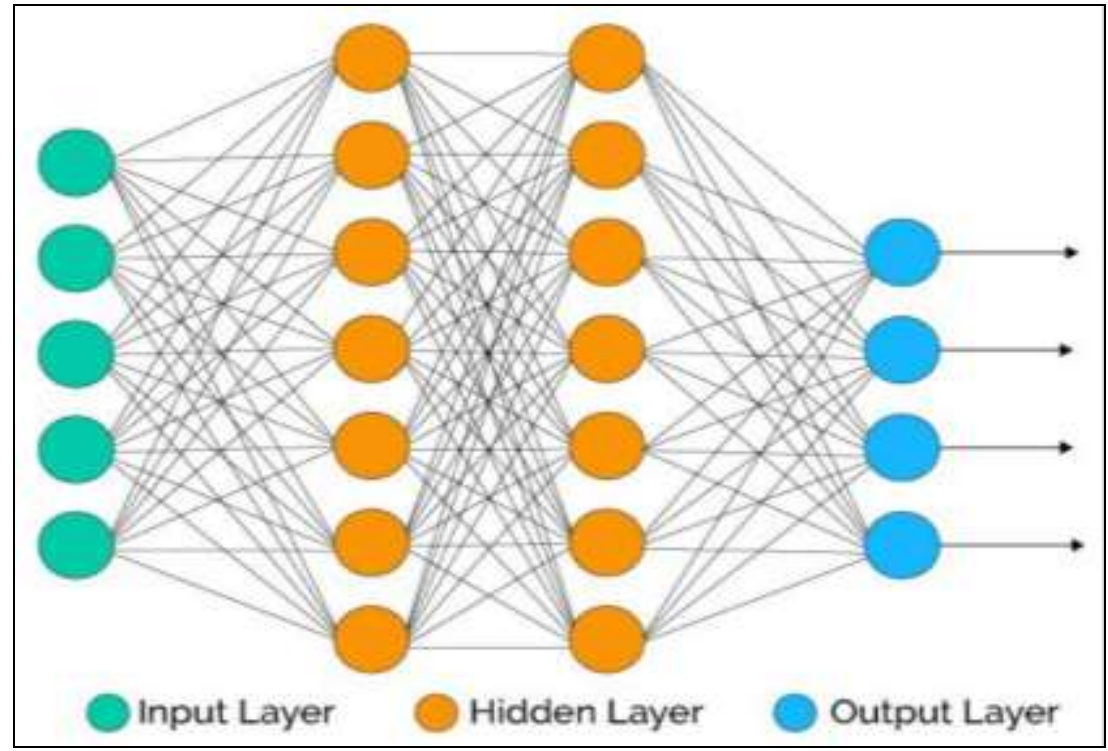

Fig 3: Deep learning [30]

From Fig. 3. The 1st layer is hidden layer for edge detection, the next layer is detecting a combination of edges followed by identifying features and combining all these layers, at last, output layer is identifying the combination of features that recognizes the object.

Deep learning (DL) is a specific form of machine learning (ML). The workflow of ML begins with the related features where it extracts manually from images and these features are used to generate a model which classify objects within the image, while deep learning (DL) extracts significant feature automatically from images and it performs "End-to-End" (E2E) learning- where model fetches raw data and automates the classification. In DL if the range of data increases, granular learning converges and improves accuracy whereas in ML training data should be specific otherwise the model reaches over fitting.

\section{Materials and Methods}

\subsection{The Channel of a Diagnostic Technique to Detect Osteoporosis}

The quantitative diagnostic imaging-modality tools follow general channels to detect Osteoporosis which are divided into 5- stages: 1) Acquisition of image 2) Pre-Processing 3) Segmentation method i.e. sub-image (ROI), 4) Extraction of feature, and 5) Classification (as shown in Fig. 4). In stage-1, Images are acquired (1) using dedicated imaging modalities and while capturing the image degradation occurs, therefore pre-processing of the image is done and in stage 2 the quality of the image can be improved by suppressing surplus deformation or image-feature enhancement is done by preprocessing method indicates transforming the raw-data prior fed to deep learning or the machine learning method ${ }^{[8]}$. The ROI-bone image segmentation depends either on manually, semi-automatic, or complete automate segmentation of the method is applied in the 3rd stage. In the 4 th stage, numerous feature-extraction techniques are considered to extort noteworthy features is taken from segmentation of ROI -bone image (cortical and trabecular bone character) features are used to diagnose nonosteoporotic or osteoporotic. The stage- 5 works on classifiers based on scores ( $Z$ and $T$ ) are computed from standard reference-values or use of ML-technique for classifying the control and osteoporotic subject.

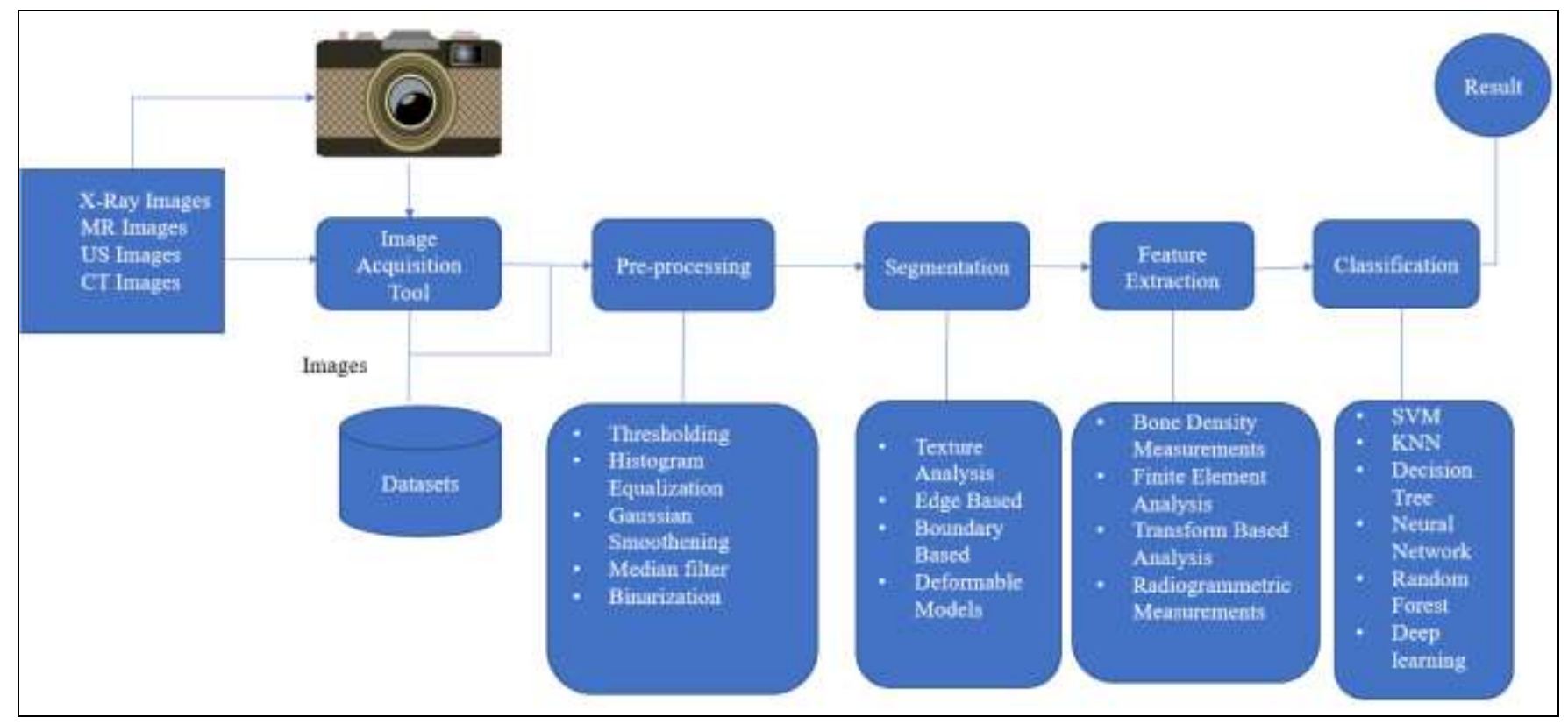

Fig 4: Channel to detect Osteoporosis ${ }^{[31]}$ 


\subsection{Quantitative Imaging Modalities to Diagnoise} Osteoporosis

The numerous Image Acquisition Tools (IAT) ${ }^{[8,9]}$ used in the medical field for detecting osteoporosis and fracture-risk prediction as shown in Fig. 5 and Table -I.

The fine-tuning and transfer learning showed better performance of deep Convolutional neural network (DCNN) for Osteoporosis screening in Dental Panoramic Radiographs (DPRs) images and by applying the mapping technique of Gradient -weighted activation class depends on features of image in right border also lower left of mandibular showed differences. The 4 groups used in DCNN Model - threeConvolutional layer (CNN3), Visual Geometry Group (VGG16), the transfer learning (VGG-16_TF) and fine-tuning by the transfer learning model(VGG-16_TF_FT) ${ }^{[1]}$ accord better accuracy ${ }^{[10]}$. These outcomes were used for screening an automatic osteoporotic patient with less training datasets. The hand radiograph (HR) classification showed Osteoporosis on the 2nd Metacarpal cortical-percentage. Segmentation was used to identify which specific pixels belong to the second metacarpal PA (Poster Anterior) x-ray images using Fully Convolutional Network-8(FCN-8) CNN with accuracy (94.8\%). The LeNet Convolutional Neural Network (LeNetCNN) used to classify Laterality with accuracy (99.62\%), Specificity (100\%) and Sensitivity (99.3\%). The overall accuracy is shown in ${ }^{[11]}$. The formation of the CNN series showed an accurate classification of an osteoporotic and control subject. In addition to this, $\mathrm{CNN}$ can generate accurate image adaptation rely on vertical and laterality alignment.

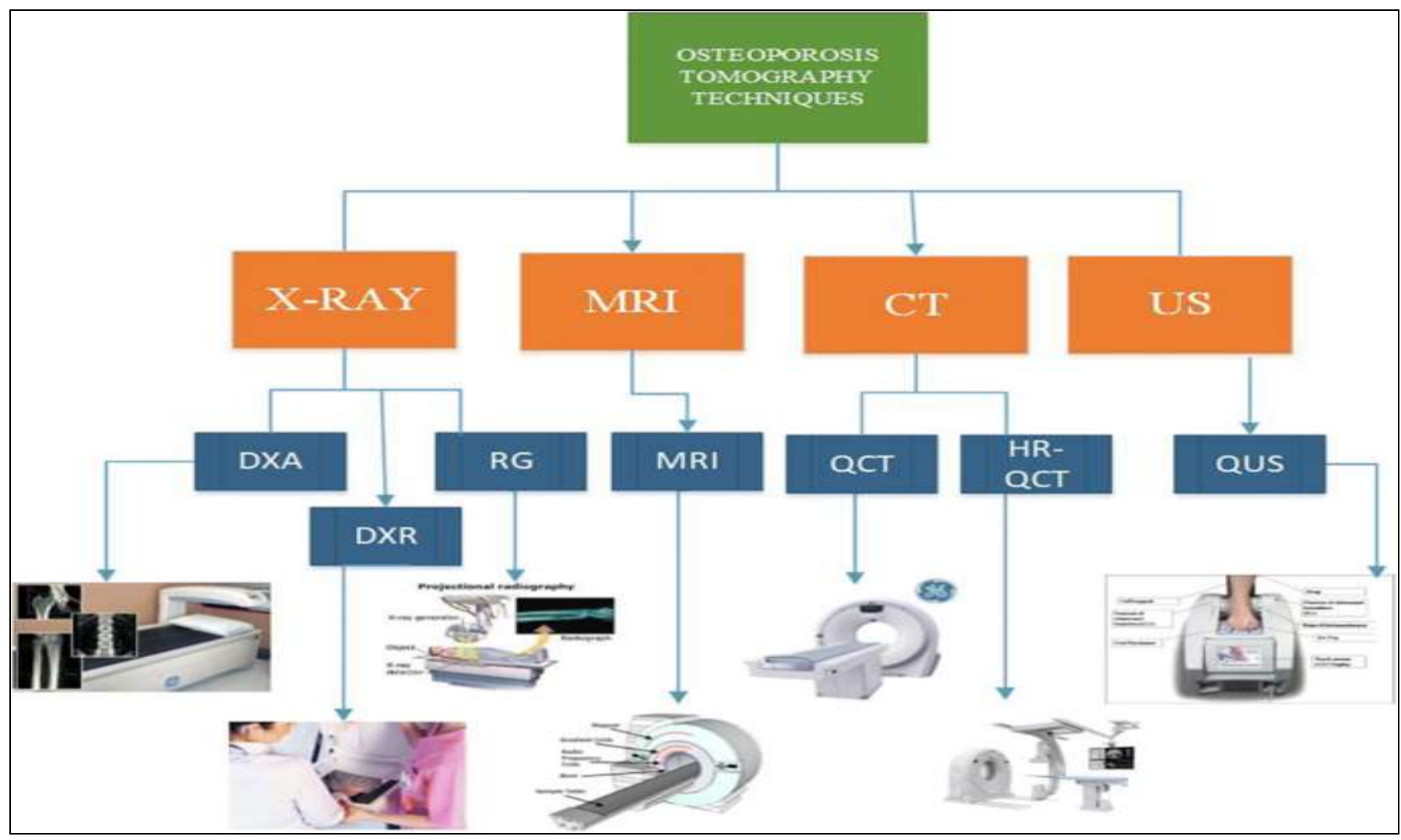

Fig 5: Imaging Modalities to diagnose Osteoporosis ${ }^{[31]}$

Table I: Performance parameters comparison for Example 1

\begin{tabular}{|c|c|c|c|c|c|}
\hline Sl. No & Image Modality & ROI & Feature Extraction & Advantages & Dis-Advantages \\
\hline 1. & $\begin{array}{l}\text { DXA (Dual X-ray } \\
\text { Absorptiometry) }\end{array}$ & $\begin{array}{l}\text { - Whole Body } \\
\text { - } \quad \text { Fore-arm } \\
\text { - } \quad \text { Lumbar Spine }\end{array}$ & $\begin{array}{l}\text { The ratio of Bone } \\
\text { Mineral Content(BMC) } \\
\text { to the total area is } \\
\text { measured by BMD }\end{array}$ & $\begin{array}{l}\text { - } \\
\text { is low } \\
\text { - } \\
\text { Gold Standard } \\
\text { Scanning time is } \\
\text { low }\end{array}$ & $\begin{array}{l}\text { Unable to define Micro- } \\
\text { architecture of bone } \\
\text { Very Expensive } \\
\text { Equipment availability is } \\
\text { very low } \\
\text { areal measurement gives } \\
\text { different for bone-sizes and } \\
\text { values }\end{array}$ \\
\hline 2. & $\begin{array}{c}\text { DXR(Digital X-ray } \\
\text { Radiogrammetry) }\end{array}$ & $\begin{array}{l}\text { Meta-carpal bones(Hand- } \\
\text { bones) }\end{array}$ & $\begin{array}{l}\text { BMD is extracted from } \\
\text { Radiogrammetry along } \\
\text { with cortical (porosity) }\end{array}$ & \begin{tabular}{|l} 
- Alternative for low \\
economy(Cost- \\
Effective) \\
No biasing human \\
error \\
Correlation is Good \\
with DXA
\end{tabular} & $\begin{array}{l}\text { Unable to compute } \\
\text { Volumetric BMD } \\
\text { Texture of Trabecular is } \\
\text { not considered }\end{array}$ \\
\hline 3. & Radiography & $\begin{array}{l}\text { Lumbar spine, hip, } \\
\text { calcaneum, dental and } \\
\text { forearm }\end{array}$ & \begin{tabular}{|l|} 
Texture \\
Analysis(Connectivi \\
ty, anisotropy)
\end{tabular} & $\begin{array}{ll}\text { - } & \text { Commonly } \\
\text { available } \\
\text { - } & \text { Cost-Effective } \\
\end{array}$ & $\begin{array}{l}\text { - } \quad \begin{array}{l}\text { Radiation exposure higher } \\
\text { It generates 2D images }\end{array} \\
\end{array}$ \\
\hline
\end{tabular}




\begin{tabular}{|c|c|c|c|c|c|}
\hline & & & Radiogrammetry & Scanning is easy & \\
\hline 4. & $\begin{array}{c}\text { MRI (Magnetic } \\
\text { Resonance Imaging) }\end{array}$ & Spine, hip and Fore-arm & \begin{tabular}{|l} 
- Compute \\
Histomorphometric \\
features(connectivit \\
y and thickness)
\end{tabular} & $\begin{array}{ll}\text { Produce } \\
\text { Volumetric } \\
\text { measurement } \\
\text { Less radiation } \\
\text { exposure } \\
\text { 3D Imaging } \\
\text { Improvement in } \\
\text { trabecular bone- } \\
\text { micro architecture }\end{array}$ & $\begin{array}{l}\text { - } \quad \text { Longer Scan time } \\
\text { - } \\
\text { low } \\
\text { - } \quad \text { Very Expensive } \\
\text { Prone to motion arti-facts } \\
\text { and volume effects }\end{array}$ \\
\hline 5. & $\begin{array}{l}\text { QUS (Quantitative } \\
\text { ultrasound) }\end{array}$ & $\begin{array}{l}\text { Tibia,radius,phalanx and } \\
\text { calcaneum }\end{array}$ & $\begin{array}{l}\text { Velocity and } \\
\text { attenuation(Sound) }\end{array}$ & $\begin{array}{ll}\text { - } & \text { No radiation } \\
\text { exposure } \\
\text { Cost is low(low } \\
\text { economy) } \\
\text { - } \\
\text { Device is Portable } \\
\text { Non-invasive and } \\
\text { simple technique } \\
\text { Sound waves is } \\
\text { used to study bone }\end{array}$ & $\begin{array}{ll}\text { - } & \text { Sensitivity and } \\
\text { Precision(poor) } \\
\text { - } & \text { accuracy (poor) } \\
\text { - } & \text { Dised for specific site } \\
\text { measurements undergo } \\
\text { minor changes in their } \\
\text { measurements }\end{array}$ \\
\hline 6. & $\begin{array}{l}\text { QCT (Quantitative } \\
\text { Computed } \\
\text { Tomography) }\end{array}$ & Hip, Lumbar Spine & $\begin{array}{l}\text { Volumetric- BMD using } \\
\text { system measurement } \\
\text { Calibration phantom }\end{array}$ & $\begin{array}{l}\text { - Involve 3D- } \\
\text { geometric imaging } \\
\text { Analyze the risk- } \\
\text { fracture for } \\
\text { trabecular and } \\
\text { cortical regions } \\
\text { It gives volumetric } \\
\text { measurements }\end{array}$ & $\begin{array}{l}\text { - } \quad \text { Radiation is higher } \\
\text { whole body scanning has } \\
\text { limited access } \\
\text { Scarcity of commercially } \\
\text { existing software } \\
\text { programming } \\
\text { - } \\
\text { Artifacts causes variations } \\
\text { WHO - norm do not apply! }\end{array}$ \\
\hline 7. & \begin{tabular}{|c|} 
pQCT(Peripheral \\
Quantitative Computed \\
Tomography) \\
\end{tabular} & Tibia, Distal Radius & $\begin{array}{l}\text { Trabecular density and } \\
\text { cortical (architecture) }\end{array}$ & $\begin{array}{l}\text { It is suitable for } \\
\text { peripheral quantity }\end{array}$ & $\begin{array}{l}\text { - Very Expensive } \\
\text { - Radiation dose is high }\end{array}$ \\
\hline
\end{tabular}

\section{Literature Review}

Recurrent Neural Network (RNN) is used to detect and segment Osteoporosis especially the spine and produces image representation of bone density. It has an excellent implementation in the medical-image analysis together with Orthopaedic issues. The practicability of RNN was evaluated and examined using two methods- transfer learning and training from scratch. The analysis reviewed that RNN can utilize image data of DXA gives prediction which differs from the standardized method used in the clinic. The ample potential architectural and with less quantity of data, their factual possibilities are outspread ${ }^{[12]}$. The study shows that pre-trained RNN provides best fracture risk prediction and reinforces the emphasized advantage of transfer learning i.e., larger datasets and faster training speed. Computer-Assisted Diagnosis (CAD) base DCNN is used to detect Osteoporosis of panoramic X-ray images compared with the diagnosis of Oral and Maxillofacial radiographs. The CAD system was used to examine panoramically-ray images- Multicolumn DCNN (MC-DCNN), (Augment SC-DCNN) Single-Column DCNN by means of Data Augmentation as well as (SCDCNN) Single-Column DCNN, all these systems were evaluated using Receiver Operating Characteristic (ROC)curve as shown in table1 and these System help to assist dentist for the prior diagnosis of Osteoporosis ${ }^{[13]}$.

The CNN approach ${ }^{[14]}$ was used to automate the condition of bone CT image by decreasing the number of patients. The technique includes Mark Segmentation (MS-net) and Bone Conditions Classification Network (BCC-net) where mark segmentation goal is to place the position and ROI-seg (Region of interest -segmentation), the BCC-net cluster the condition of bone through features. The trained MS network gives marking on input CT (Computed Tomography) image which leads to segmentation and the BCC network finds the probable value of healthy, Osteopenia, and osteoporotic by segmenting input CT image. The outcome of these network aid radiologists for prelude quality analysis of bone-condition. A Multitask approach used in DNN to pretrained Image-Net for DPR images and enhancing the accuracy to diagnose osteoporosis [15]. The task of Alex-Net is pre-trained on Image-Net used for fine-tuning and input (Patient)classification are profuse partial patches, extraction of DPR images, and the learned features (dental-data) are used for osteoporosis detection. So, Octuplet Siamese Network (OSNet) is the highest feature and 8th trained- ROI DPR-image categorization. The cross-validation is leave-1out to achieve higher accuracy as shown in Table-1.Artificial neural network (ANN) is a model used to improve the score of OSTA-(Osteoporosis Self-Assessment Tool for Asian's) and was created using female attributes ${ }^{[16]}$ as an input (age, weight) with output femoral neck (T-score). The ROC (Receiver Operating Characteristic) Curve examined by integrating OSTA Score and ANN for screening osteoporosis with improved performance as shown in the table2. Fifteen Classifiers with randomized (200) cross-validation datasets were used. The feature vector of 5 femoral regions(femoral head, greater trochanter, inter-trochanter, neck, and ward) in addition to BMD, fracture risk -assessment tool (FRAX) score, and attributes (age, weight, and height).Out of 15Classifiers only 3 -classifiers(the linear discriminant, logistic regression, and boosted trees) gave the best results(sensitivity$71 \%$ and specificity-83\%)for detecting osteoporotic fracture [17]. The MRI and FRAX autonomously showed the best results for recognizing osteoporotic fracture. The bone-quality improvement through mapping of ANN. Addition of Teriparatide composition (T-PD) for not only remodeling BMD (Bone Mineral Density), also BSI (Bone-Strain-Index), TBS (trabecular-bone-score), which strengthens the bone that and minimizes the risk of fracture. The BSI seems to be a sensitivity index of the TPD effect ${ }^{[18]}$. ANN is a valid tool for 
investigation in medical applications.ANN is better than logistic regression [LR] for better accuracy as there is a greater number of inputs, discrete parameters, and optimal technique to diagnose osteoporosis. The result of ANN with respect to AUC is more than LR as shown in the table 2 with $\mathrm{P}$-value $=0.034^{[19]}$. Fuzzy logic calculations can be used with LR enhanced the best clinical method put into practice. In order to integrate with the information-management system for better analysis. The software package of ANN is an efficient approach for an accurate estimate of segmental and BMD (total) ${ }^{[20]}$. The input attributes: weight, sex, age, BMI, and height beside with segmental reference and BMD (totalvalues) are fed to ANN (multilayered-input layer) and quantifiable approximation of [BMDlegs, BMDarms, BMDpelvis, BMDspine, and BMDtotal] is generated in the output-layer. ANN model shows a potential approach for evaluating BMD (total-values) and, segmental using statistical values prove the best model to a diagnosis of osteoporosis. To detect Osteoporosis impulse-response test was done on tibial bone using Lab-VIEW. The record of analog-signal was studied in freq-domain (frequency) [21]. The vibration generated by natural frequency was a considerable decline in Osteoporosis which shows a reduction in bone strength automatic and mass in bone- minerals. In a recent study, osteoporosis detection was found high-priced and requirement of the virtuoso tool. The method used in this study was easy to use and economically less price. The trend of AI -ML effort linked to the spine include vertebral localization and images of X-ray(discs) ${ }^{[22]}$, segmentation of image(ROI), CAD, clinical practice prediction and difficulties, information management the system, bio-mechanics, Retrieving the content of the image, and motion-study. The application of AI in Medical-science secures and authenticates the data and provides the privacy community of real domain appliances. The machine learning model used to evaluate Osteoporosis by HU (Hounsfield units) of surgical on lumbar -CT pairing with QCT-data. MRA (Multiple Regression Algorithm) uses a Tscore prediction of 3-independent attributes (sex, age, and HU -Vertebral body on predictable CT) combining with T-scoreQCT. The algorithm of logistic regression helped to assist non- osteoporotic vertebra. With the monitoring of machine learning tools Python, the Tensor flow helped the programmer to interface with easier use ${ }^{[23]}$. The Algorithm helps to predict data of QCT of T-scores. The training data sets of this model segregated the lumbar vertebra into 2 classifiers of the nonosteoporotic, osteoporotic spine with better accuracy as shown in the table 2 . In testing-phase, the datasets were 40 vertebrae used to improve the accuracy (92.5\%), learning rate(0.0001),(precision, 0.939; recall, 0.969; F1 score, 0.954; area under the curve, 0.900$)$. The dynamic method for selection of features, categorization, and detection by using supervised learning methods- Nearest Neighbours (NNs), Support Vector Machines (SVMs) with improved accuracy of $95 \%$ as shown in the table $2^{[24]}$. Further yields cost-effective and robust in nature. The 4 attributes were used for prediction of Osteoporosis (height, weight, age, and sex,). To discriminate non-osteoporosis and osteoporosis, 20 ML-Technique were applied depending on the popularity in the biomedical engineering field. The 20 classifiers were validated using 10fold cross-validation and the results were systematically predicted to avoid further testing. The analyses were standardized and diminished to two attributes (age and weight) which yielded similar results ${ }^{[25]}$. ML classifiers were designed in WEKA (benchmark of ML) are tested using 10fold cross-validation, training datasets and splitting percentage inclusion and exclusion feature selection. The comparison of the result is done in terms of execution time, classified instances, mean absolute values, and kappa statistics evaluation inclusion and exclusion of feature selection ${ }^{[26]}$. The overall study propose IBK (training and testing set) gives better result exclusion feature- selection, where these techniques [IBK, LMT, J48, JRip, SMO, and bagging] gives an appreciable result of inclusion feature selection. Texture-Characterization of good-quality bone is necessary for Osteoporosis Identification. The Gray Level Cooccurrence Matrix (GLCM), Local Binary Pattern (LBP), Law's, and so on are the standard methods used for texture feature extraction. A collate between deep- features extraction from CNN adjacent conventional features ${ }^{[27]}$. The outcome of this study demonstrates that deep-features have more distinguishing power with respect to classifiers training on them constantly surpass one's training on conventional features.

\section{Results \& Discussions}

The majority of the Research-work is carried out on osteoporosis detection to circumvent the risk of bone fracture however it is highly affected to trabecular bone i.e., soft and spongy bone rather than compact cortical hard bone. The performance of a system is compared with dissimilar the technique utilized in the current trend are mentioned in Table II.

Table II: Performance Comparison with different methods

\begin{tabular}{|c|c|c|c|c|c|c|}
\hline Model & $\begin{array}{c}\text { Imaging } \\
\text { Modality } \\
\end{array}$ & Human Site & $\begin{array}{l}\text { Classification Module/ } \\
\text { Pre-Trained Networks }\end{array}$ & Validation & $\begin{array}{c}\text { Accuracy } \\
(\%)\end{array}$ & $\begin{array}{l}\text { Auc } \\
(\%)\end{array}$ \\
\hline 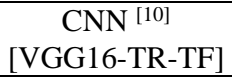 & X-RAY & $\begin{array}{c}\text { DPR } \\
\text { (Dental panoramic) }\end{array}$ & VGG16-TR-TF & 5-fold cross & 84 & 85.8 \\
\hline $\mathrm{CNN}^{[11]}$ & X-RAY & Hand & LeNet- based CNN & & 93.9 & - \\
\hline RNN [12] $^{[12}$ & MRI & lumbar spine & 10-fold cross & & & \\
\hline $\begin{array}{c}\mathrm{DCNN}^{[13]} \\
{[\text { (SC-DCNN) }} \\
\text { SC-DCNN } \\
\text { (Augment) } \\
\text { (MC-DCNN] }\end{array}$ & X-RAY & Dental panoramic & 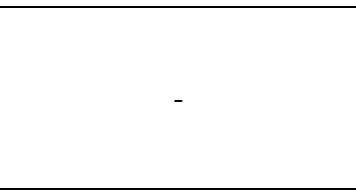 & 200 (testing) & $\begin{array}{c}98 \\
98.5\end{array}$ & $\begin{array}{l}97.63 \\
99.91 \\
99.87\end{array}$ \\
\hline $\mathrm{CNN}^{[14]}$ & $\mathrm{CT}$ & lumbar vertebra & $\begin{array}{l}\text { BCC-net } \\
\text { MS-net }\end{array}$ & $\begin{array}{l}\text { 7- fold } \\
\text { 2- fold }\end{array}$ & 76.5 & 91.67 \\
\hline $\begin{array}{l}\text { MULTI-TASK } \\
\text { SCHEME }^{[15]}\end{array}$ & X-RAY & Dental panoramic & OSNet & $\begin{array}{c}\text { Leave-one-out } \\
\text { cross-validation }\end{array}$ & $92.59 \%$ & - \\
\hline $\begin{array}{c}\text { ANN }{ }^{[16]} \\
\text { OSTA-Score }\end{array}$ & $\begin{array}{l}\text { X-RAY \& } \\
\text { DXA }\end{array}$ & Femoral Neck & ANN & 10-fold cross & $\begin{array}{l}78.8 \\
78.3\end{array}$ & - \\
\hline $\mathrm{ANN}^{[17]}$ & MRI & Femoral Neck & linear discriminant, logistic & 23- fold & 71 & - \\
\hline
\end{tabular}




\begin{tabular}{|c|c|c|c|c|c|c|}
\hline & & & $\begin{array}{l}\text { regression, and boosted } \\
\text { trees }\end{array}$ & & & \\
\hline $\mathrm{ANN}^{[19]}$ & X-RAY & Vertebral & $\begin{array}{c}\text { ANN } \\
\text { logistic regression }\end{array}$ & $\begin{array}{c}\text { 30-osteoporosis } \\
\text { 35-non- } \\
\text { Osteoporosis } \\
\text { 54-Prediction } \\
\end{array}$ & 95.8 & $\begin{array}{c}95 \\
87.0\end{array}$ \\
\hline $\mathrm{ANN}^{[20]}$ & DXA & $\begin{array}{l}\text { Arms, legs, spine, pelvis, } \\
\text { and total }\end{array}$ & $\begin{array}{l}\text { Demographic Variables } \\
\text { (Age, weight, height, } \\
\text { lifestyle etc) }\end{array}$ & $\begin{array}{l}200 \text { cross } \\
\text { validations }\end{array}$ & $\begin{array}{c}99.78, \\
99.04, \\
99.19, \\
99.89, \& \\
99.9\end{array}$ & - \\
\hline $\begin{array}{l}\text { Feed-forward back } \\
\text { propagation }{ }^{[21]}\end{array}$ & $\mathrm{CT}$ & tibial bone & ANN & 70 & - & - \\
\hline $\begin{array}{c}\text { Multiple } \\
\text { Regression } \\
\text { Algorithm [23] }\end{array}$ & $\mathrm{CT}$ & Lumbar vertebrae & Logistic Regression & $\begin{array}{c}\text { Training-158 } \\
\text { Testing-40 }\end{array}$ & 92.5 & 90.0 \\
\hline GLCM $^{[24]}$ & X-ray & Calcaneus & SVM, NN & 30-fold & 95 & - \\
\hline $\begin{array}{c}\text { Features of BMD } \\
\text { [25] }\end{array}$ & DXA & $\begin{array}{l}\text { Osteoporotic site (hip, } \\
\text { spine and arm) }\end{array}$ & Multilayer perceptron & 10-fold & 71.51 & - \\
\hline $\begin{array}{l}\text { Machine Learning } \\
\text { Technique }\end{array}$ & - & $\begin{array}{l}\text { family history, blood } \\
\text { calcium, vitamin, and } \\
\text { phosphorus. }\end{array}$ & LMT [logistic model tree] & 10-fold & 87.14 & - \\
\hline Deep Features ${ }^{[27]}$ & X-ray & Calcaneus & Random Forests & $\begin{array}{l}\text { Training-116 } \\
\text { Testing-58 }\end{array}$ & 79.3103 & 0.85 \\
\hline
\end{tabular}

\section{Conclusion}

On boosting follow-up care and identifying personnel with higher risk of fracture requires prompt treatment, coped up with care can enhance the cost-effective management of osteoporosis, dampening downstream costs. However, in the recent medical diagnostic field, DXA is not popularly accessible and it is not cost-effective with several disadvantages and it is not an absolute solution. So, it is necessary to construct a diagnostic system to fulfill the necessities incorporating reliability, ease of availability, costeffectiveness, and clinical acceptance. In this review study CAD-system vision-based for automatic detection and diagnosis of osteoporosis which inclusion of X-rays, DXA scans, MRIs, and CT-scan of vertebrae were surveyed. The channel to detect Osteoporosis includes acquisition of image, pre-processing, segmenting with respect to ROI, extracting features and lastly the classifiers using machine learning technique. Further, it is observed that texture analyses of trabecular micro architecture using ML based techniques are used to improve the sensitivity, specificity, F1-score and accuracy. The advantage of using ML Technique is selflearning because no further preprocessing is required. However, the standardization of dataset is needed for researchers to assist a universal paradigm. The future directions of research could be concerned to X-ray diagnostic significant tool of low-cost, extensive use and ease of availability. The vision of deep learning model needs large amount of data and the machine in DL learn features automatically from the input data.

\section{Acknowledgement}

This work was supported by Dr. A Ramalingaiah M.S (ortho) Orthopedic Doctor, Bangalore. No. 271 Abhilasha Orthopaedic Hospital 5th Block 100ft Rd Banashankari 3rd Stage Bangalore -560085 .

\section{References}

1. https://www.ncbi.nlm.nih.gov/books/NBK45504/.

2. Nutrient Requirements and Recommended Dietary Allowances for Indians: A Report of the Expert Group of the Indian Council of Medical Research; 2009. Hyderabad: National Institute of Nutrition; Indian
Council of Medical Research. Identification of risk groups for osteoporosis, a general review," Biomedical engineering online 2018;17(1):12 29 Jan. doi:10.1186/s12938-018-0436-1.

3. Nam, Kyoung Hyup, et al., "Machine learning model to predict osteoporotic spine with hounsfield units on lumbar computed tomography, Journal of Korean Neurosurgical Society 2019;62(4):442.

4. Iliou, Theodoros, Christos-Nikolaos Anagnostopoulos, and George Anastassopoulos, "Osteoporosis detection using machine learning techniques and feature selection, International Journal on Artificial Intelligence Tools 2014;23(05):1450014.

5. Tomita, Naofumi, Yvonne Y Cheung, Saeed Hassanpour. "Deep neural networks for automatic detection of osteoporotic vertebral fractures on CT scans, Computers in biology and medicine 2018;98:8-15,

6. Yu, Xinghu, Chao Ye, Liangbi Xiang. "Application of artificial neural network in the diagnostic system of osteoporosis," Neurocomputing 2016;214:376-381.

7. Areeckal AS, Kocher M, SDS. "Current and Emerging Diagnostic Imaging-Based Techniques for Assessment of Osteoporosis and Fracture Risk, IEEE Reviews in Biomedical Engineering 2019;12:254-268 doi: 10.1109/RBME.2018.2852620.

8. Wani, Insha Majeed, Sakshi Arora. "Computer-aided diagnosis systems for osteoporosis detection: a comprehensive survey," Medical \& Biological Engineering \& Computing 2020, 1-45,

9. Lee, Ki-Sun, et al., "Evaluation of Transfer Learning with Deep Convolutional Neural Networks for Screening Osteoporosis in Dental Panoramic Radiographs," Journal of Clinical Medicine MDPI, 2020;392:9

10. Tecle, Nahom et al., "Convolutional Neural Network for Second Metacarpal Radiographic Osteoporosis Screening, The Journal of Hand Surgery 2020;45(3):75181.

11. Atheel Sabih Shaker, "Detection and Segmentation of Osteoporosis in Human Body using Recurrent Neural Network", International Journal of Advanced Science and Technology 2020;29(02):1055-1066.

12. Eason G, Noble B, Sneddon IN. "On certain integrals of 
Lipschitz-Hankel type involving products of Bessel functions," Phil. Trans. Roy. Soc. London, 1955;A247:529-551. (References).

13. Lee, Jae-Seo, et al., "Osteoporosis detection in panoramic radiographs using a deep convolutional neural networkbased computer-assisted diagnosis system: a preliminary study," Dentomaxillofacial Radiology 2019;48(01):2017034.

14. Tang, Chao, et al., "CNN-based Automatic Detection of Bone Conditions via Diagnostic CT Images for Osteoporosis Screening," arXiv, preprint arXiv:1910.06777, Oct 142019.

15. Yua, Sijia et al., "Multi-task Osteoporosis Pre-screening Using Dental Panoramic Radiographs with Feature Learning," Smart Health, Elsevier B.V, 2019.

16. Meng Jia, et al., "Artificial neural network optimizes self-examination of osteoporosis risk in women," Journal of International Medical Research 2019;47(7):3088-3098. July

17. Ferizi, Uran, et al., "Artificial intelligence applied to osteoporosis: a performance comparison of machine learning algorithms in predicting fragility fractures from MRI data," Journal of Magnetic Resonance Imaging, 2019;49(4):1029-1038.

18. Messina, Carmelo et al., "Artificial neural network analysis of bone quality DXA parameters response to teriparatide in fractured osteoporotic patients," Plos one, 2020;15(3):e0229820.

19. Yu, Xinghu, Chao Ye, Liangbi Xiang. "Application of artificial neural network in the diagnostic system of osteoporosis," Neurocomputing 2016;214:376-381.

20. Abdel-Mageed, Samir M, Amani M Bayoumi, Ehab I Mohamed. "Artificial neural networks analysis for estimating bone mineral density in an Egyptian population: towards standardization of DXA measurements," American Journal of Neural Networks and Applications 2015;1(3):52-56.

21. Tejaswini E, Vaishnavi P, Sunitha R. "Detection and prediction of osteoporosis using impulse responsetechnique and artificial neural network, In 2016International Conference on Advances in Computing, Communications and Informatics (ICACCI). 2016, 15711575, IEEE,

22. Ferizi, Uran, Stephen Honig, Gregory Chang. "Artificial intelligence, osteoporosis and fragility fractures, Current opinion in rheumatology 2019;31(4):368-375.

23. Galbusera, Fabio, Gloria Casaroli, Tito Bassani. "Artificial intelligence and machine learning in spine research, JOR spine 2019;2(1):e1044.

24. Nam, Kyoung Hyup, et al., "Machine learning model to predict osteoporotic spine with hounsfield units on lumbar computed tomography, Journal of Korean Neurosurgical Society 2019;62(4):442.

25. Bhattacharya Sayantan. et al., "Computer Based Automatic Detection and Classification of Osteoporosis in Bone Radiographs,",6th International Conference on Signal Processing and Integrated Networks (SPIN), IEEE, 2019, 1047-1052.

26. Delen, Dursun. "A comparative analysis of machine learning techniques for student retention management, Decision Support Systems 2010;49(4):498-506.

27. https://www.genetrace.com/tests/bone-health-andosteoporosis-panel/.

28. https://www.simplilearn.com/tutorials/deep-learningtutorial/deep-learning-with-python
29. https://heartbeat.fritz.ai/a-2019-beginners-guide-to-deeplearning-part-1-facff72a6097

30. https://www.pnas.org/content/116/4/1074.

31. https://link.springer.com/article/10.1007/s11517-02002171-3 